J. Asiat. Soc. Bangladesh, Sci. 46(1): 27-35, June 2020

\title{
TEMPORAL DISTRIBUTION AND ABUNDANCE OF MOSQUITO VECTORS IN DHAKA CITY
}

\author{
M.G. SHAROWER ${ }^{1 *}$, M.A. LATIF ${ }^{2}$ AND S.M. UDDIN ${ }^{3}$ \\ ${ }^{I}$ Department of Public Health, North South University, Bashundhara, \\ Dhaka-1229, Bangladesh \\ ${ }^{2}$ Department of Entomology, Sher-e-Bangla Agricultural University, \\ Sher-e-Bangla nagar, Dhaka-1207, Bangladesh \\ ${ }^{3}$ Dhaka North City Corporation, Gulshan-2, Dhaka-1212, Bangladesh
}

\begin{abstract}
Species of Anopheles, Aedes and Culex mosquitoes showed that Anopheles gambiae s.s had the highest number (43.5\%) out of the three malaria vectors (viz. Anopheles gambiae, An. arabiensis and An. funestus). For Aedes and Culex species, Aedes aegypti (37.6\%) and Culex fatigans $(37.1 \%)$ had the highest prevalence out of their sibling species. Temperature and rainfall were highly correlated with the abundance of mosquito vectors. It was observed that the rainy season (March to October) recorded the highest number (Total 11 specie) of mosquito vectors collected with the peak (Aedes aegypty, 140) in the months of July (932) and August (976) while the lowest (333) collection was in the dry season (November to February) with lowest (333) in the month of February when there was little or no rains.
\end{abstract}

Key words: Temporal distribution, Abundance, Mosquito vectors

\section{Introduction}

In warm and tropical climatic regions of the world, climatic factors have been associated with relative mosquito abundance and transmission of mosquito borne infections. Approximately half of the world's population is at the risk of malaria and an estimated 243 million infected cases resulted in nearly 86300 deaths in 2008 (WHO 2009). In SubSaharan Africa, $91 \%$ of malaria was detrimental and caused death. Malaria was estimated by calculating the result in an annual loss of $85 \%$ of the deaths amongst children below five years (WHO 2010). In addition $40 \%$ of all the public health spending is related to malaria (Haque et al. 2010). Out of all the diseases, such as malaria, filariasis, dengue and yellow fever caused by mosquito vectors, malaria is the most important tropical and parasitic disease in the world. Malaria alone accounts for up to $25 \%$ of hospital attendance, with young children under five years accounting for about $40 \%$ in Bangladesh (WHO 2012). Filariasis also has been shown to be a public health problem in Bangladesh, particularly in the Dhaka City Corporation (Ahmed et al. 2009). Studies

*Corresponding author: sharower_bau@yahoo.com 
throughout the world have shown that incidence of malaria and other related diseases associated with mosquito vectors are linked to the pattern of rainfall, temperature and humidity (Briet et al. 2008). Rainfall is considered to be a major factor influencing malaria outbreak in Bangladesh (Haque et al. 2007) and a causal relationship between rainfall and malaria transmission is well recognized (Thomson et al. 2005). In the highland of Kenya, malaria cases increased by 1.4 to $10.7 \%$ per month for each $10 \mathrm{~mm}$ increase in monthly rainfall (with 2-3 months lag) (Hashizume et al. 2009). Natural climatic disasters such as floods and cyclones may also have significant relationship with malaria outbreaks (Lindsay and Birley 1996). Temperature, rainfall and humidity have been widely associated with the dynamics of malaria vector population and therefore with the spread of the disease.

However, at the local scale, there is lack of a systematic quantification of these factors of malaria transmission (Githeko and Ndgwa 2001). In East African Highland the research findings of Zhou et al. (2004) revealed that $1^{\circ} \mathrm{C}$ temperature increase in minimum temperature having a lag of time of $1-2$ months and $1^{\circ} \mathrm{C}$ increase in maximum temperature with a lag time of 2-5 months led to an $80-95 \%$ increase in the number of malaria outpatients. Meteorological factors are important drives of malaria transmission. Ambient temperature plays a major role in the life cycle of the malaria vector. Temperature between 15 and $40^{\circ} \mathrm{C}$ and humidity between 55 and $80 \%$ are suitable for the completion of the Plasmodium falciparum and $P$. vivax malaria parasites life cycle (Zhou et al. 2004). The development of the parasite within the mosquito (sporogonic) cycle is dependent on temperature. The sporogonic cycle takes about $9-10$ days at $28^{\circ} \mathrm{C}$ but stops at temperature below $16^{\circ} \mathrm{C}$ (Lindsay and Birley 1996). The daily survival of vector is dependent on temperature as well. The suitable survivality of daily temperature is between 16 and $36^{\circ} \mathrm{C}$ and the daily survival capability drops rapidly at temperature above $36^{\circ} \mathrm{C}$. The highest proportion of vector surviving incubation period is reported at temperature between 28 and $32^{\circ} \mathrm{C}$ (Craig et al. 1999). The gonotrophic cycle which is the time between blood meals of the vector is short at higher temperatures because digestion speed increases (Haque et al. 2010). The mosquitoes survivality was prolonged at high temperature along with frequent rainfall and suitable relative humidity of at least 50 $60 \%$ by providing breeding sites to lay eggs. Relative humidity below $60 \%$ shortens the life span of the mosquito vectors (Rogers and Randolph 2006). The goal of this work is to study the effects of these climatic factors on the distribution and abundance of mosquito vectors in the study area. 


\section{Materials and Methods}

The study was carried out in some selected houses of all the 91 words of Dhaka City Corporation (both North and South) from July, 2014 to June, 2016 (two years). Adult mosquito samples were collected from the houses between 05.00 and $07.00 \mathrm{hrs}$ in the morning and 19.00 and $21.00 \mathrm{hrs}$ in the evening when the mosquitoes attracted to the light trap (CDC-LT) baited in the $\mathrm{CO}_{2}$ and pyrethrum were collected with an aspirator. Human Landing Catches (HLC) were also performed by two trained collectors (adult male volunteers) working alternatively for one hour and resting for one hour. Medical nurses provided medical supervision of the collectors. Light trap per net catches was performed using a CDC mini light trap placed adjacently and above an occupied bed net. Pyrethrum Spray Catches were performed at 7 a.m. by spraying pyrethrum for $30-45$ seconds in the room. After 10 minutes, dead and immobilized mosquitoes were collected. Two sites per location were randomly selected. In each site, three rooms were randomly chosen within a $15 \mathrm{~m}$ distance. Each night, a different sampling method was tested in each room. The houses used for mosquito collection were randomly selected close to the sites of larval habitats. Paper cups covered with netting materials, which contained cotton pad soaked in $10 \%$ glucose, were used for collection. The cups were placed in a cool box and transported to the laboratory where the mosquitoes were anaesthetized with ethyl acetate. They were sorted out and identified by morphological characteristics with the key aids of Strickland and Choudhury (1927), Giles (1968) and Koekemoer et al. (2002). Data were collected in dawn and just after sun set for maintaining lowest temperature and at noon for recording highest temperature. Aspirator was used to count the number of mosquitoes. They were later counted and recorded.

The data on minimum and maximum temperature, rainfall and relative humidity for these periods were collected from different parts of Dhaka city every month. The water from the sample sites was collected using rain barrels. A $\mathrm{pH}$ meter was used to test the $\mathrm{pH}$ level of the water.

\section{Results and Discussion}

A total number of 7,468 adult mosquito was collected in the whole year, out of which 1808 (24.2\%) were Anopheles sp., 2900 (38.8\%) Culex sp. and 2760 (36.9\%) for Aedes sp. Anopheles gambiae, An. funestus and An. arabiensis were the malaria vectors found during the survey. An. gambiae had the highest prevalence of 787 individuals (43.5\%)

followed by An. arabiensis of 616 individuals (34.1\%) and the least in number was found in case of An. funestus 405 (22.4\%) (Table 1). 


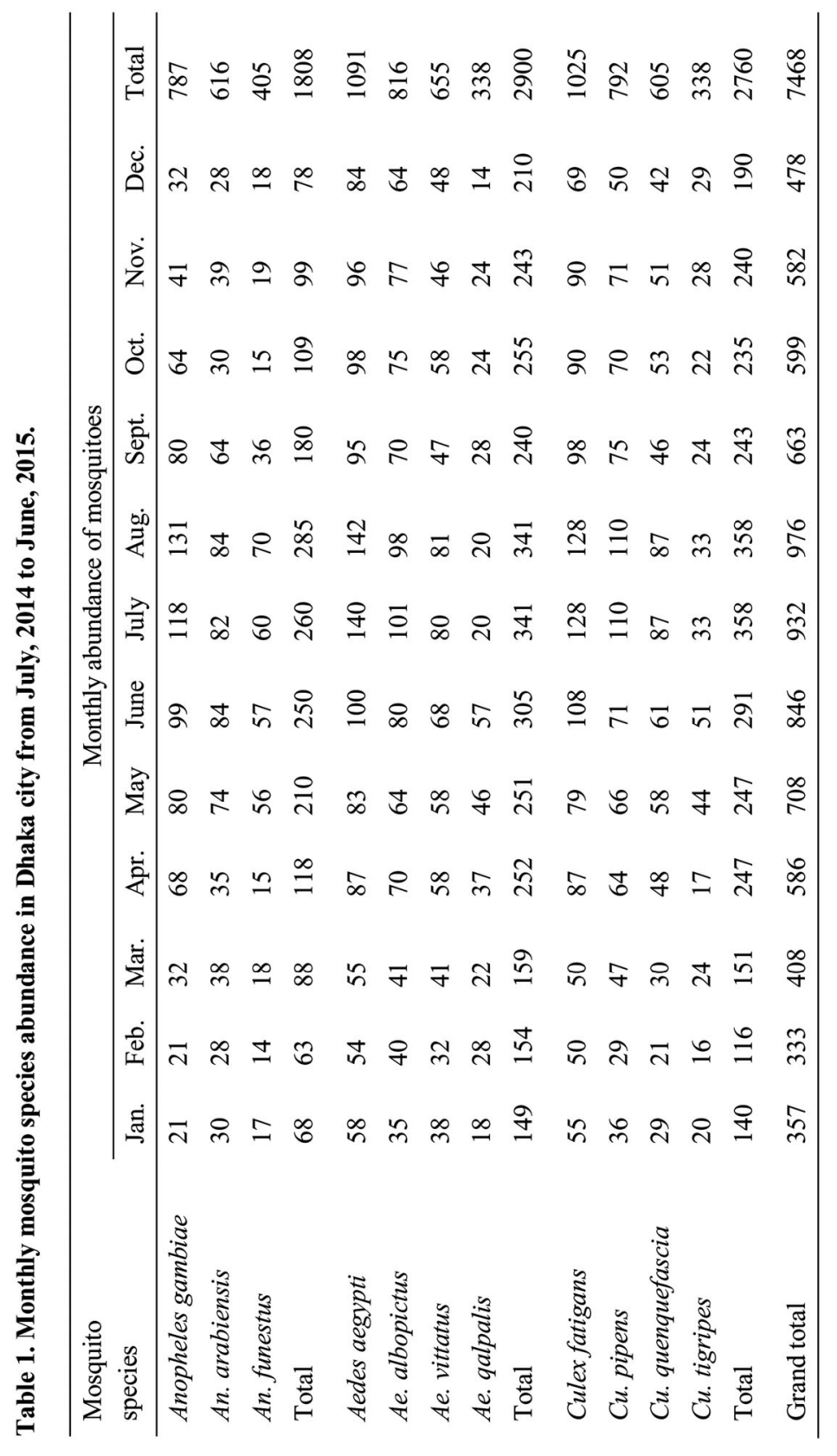


The highest numbers of mosquito species were recorded in the month of August with Aedes aegypti and Culex fatigans was found to be the most common of all the species. The distribution and abundance of the mosquito with temperature and rainfall are shown in Fig. 1.

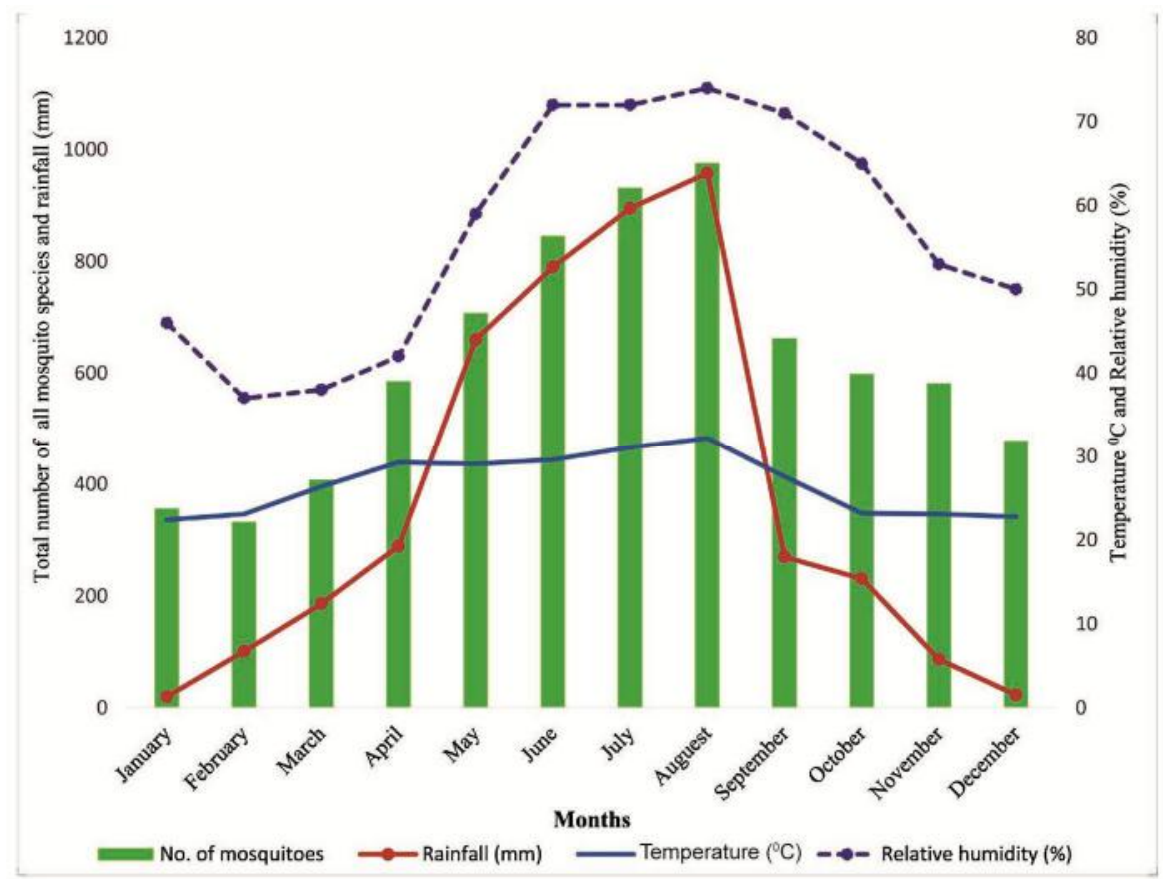

Fig. 1. Monthly prevalence of all mosquito species with temperature, rainfall and relative humidity in Dhaka city from July, 2014 to June, 2015.

Correlation coefficient between the average temperature and the total number of mosquitoes per month showed that the average temperature exhibited high correlation $\left(\mathrm{r}^{2}=0.27\right)$ with the total number of mosquito $(\mathrm{p}<0.05)$ which indicated that temperature had a significant effect on the abundance of mosquito vectors (Table 2). The correlation analysis between monthly rainfall and the total number of mosquito collected revealed that there was a very high correlation $\left(\mathrm{r}^{2}=0.79\right)$. Rainfall had a significant effect on the distribution and abundance of the mosquito vectors ( $p<0.05$, Table 2$)$. The result of the $t$ test analysis carried out on the effects of both temperature and rainfall on the monthly abundance of mosquito vectors showed that they had a significant effect on climatic factors (Table 2). 
Table 2. Analysis of correlation between the meteorological parameter and abundance of mosquito vectors $(n=12)$.

\begin{tabular}{llll}
\hline Variables & CC & p values & r2 \\
\hline Rainfall vs TNMVs & 0.85 & 0.001 & 0.79 \\
Temperature vs TNMVs & 5.02 & 2.200 & 0.27 \\
Rainfall and temperature vs TNMVs & 4.77 & 2.080 & \\
\hline
\end{tabular}

$\mathrm{CC}=$ Correlation coefficient, $\mathrm{TNMVs}=$ Total number of mosquito vectors, $\mathrm{p}<0.05$.

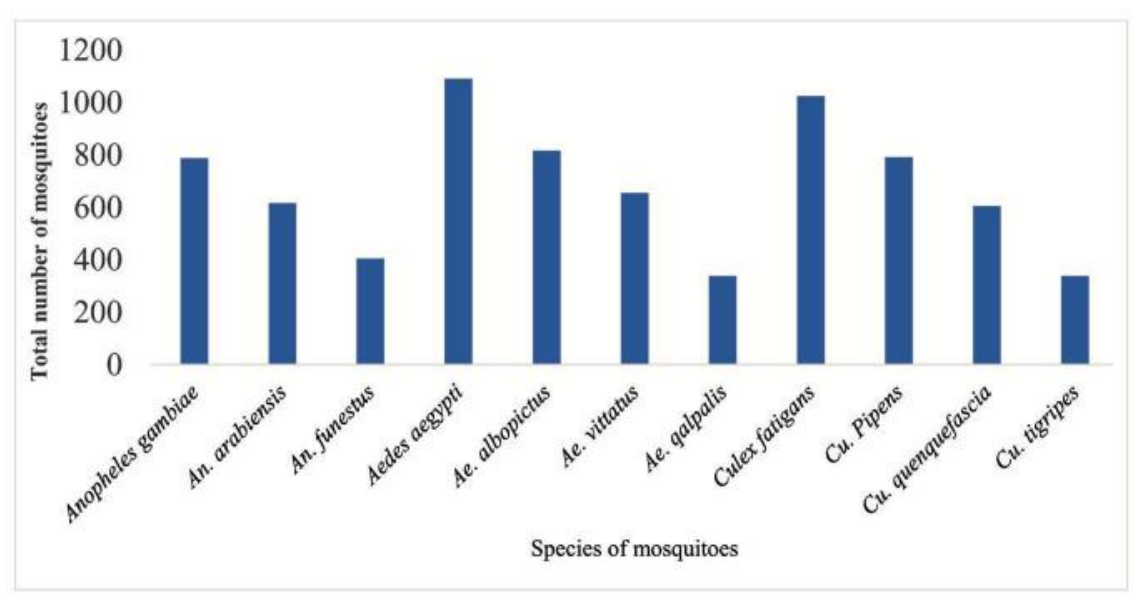

Fig. 2. Distribution pattern of all mosquito species in Dhaka city.

It is important to study the impact of weather on the transmission of malaria and other diseases associated with mosquito vectors, as global warming might change the pattern of temperature and rainfall which may directly or indirectly influence mosquito density or distribution (Wu et al. 2007). In this present study, the temperature was between 22.4 and $32.2^{\circ} \mathrm{C}$ (Fig.1) with average humidity of $56.5 \%$ (Fig. 1) which might have facilitated the higher mosquito abundance. Similar kind of results has also been previously reported on the maximum survival rate of mosquito for the related temperature and humidity (Murty et al. 2010). Thu et al. (1998), also explained in their report that humidity was one of the vital factors affecting the population density of mosquitoes and it had been observed that the temperature at $28^{\circ} \mathrm{C}$ with $55-55 \%$ relative humidity was the most appropriate condition for the elevation in mosquito density or abundance than the condition of lower temperature with higher humidity $\left(22^{\circ} \mathrm{C} / 80-85 \% \mathrm{RH}\right)$. Three main species of mosquito vectors were found, viz., Anopheles, Culex and Aedes. Among the three malaria vectors species Anopheles found, An. gambiae had the highest number (Table 1) during the rainy 
periods (May - August). For Aedes mosquitoes, four species (Table 1) were found out of which Ae. aegypti had the highest number of abundance (Table 1) while Ae. palpalis had the least number. $C x$. fatigans had the highest number (Table 1) and $C x$. tigripes had the least abundance (Table 1). This result may be as a result of the fact that their larvae can colonize and survive in almost all habitats, such as the water or barrels, drainages, tyres, pots, discarded plastics and bottles and tanks. The pattern of rainfall also affects larval habitat and vectors population size. On the basis of rainfall, increased rainfall may increase larval habitats and vector population by creating a new habitat. Excessive rain could also eliminate habitats through flooding, thus decreasing the vector population especially malaria vectors because they prefer sunlit pools of water. During the dry season, limited rainfall can also create new habitats of Anopheles gambiae, Aedes aegypti and Culex fatigans species when water in the rivers is drawn into pools, providing the perfect breeding site for a number of mosquito species, thus favouring disease transmission as also observed by Gubler et al. (2001).

The temporal change in mosquito abundance is mainly caused by rainfall. An. gambiae adults were more abundant during the rainy season than during the dry season which is consistent with the finding that the number of larval habitats was substantially higher in the rainy season than in the dry season as previously reported by Zhou et al. (2007). The lower abundance of An. funestus adults than An. gambiae was caused by the lack of suitable, long-lasting larval habitats for An. funestus because its larvae normally take three weeks to develop into adults, and An. gambiae s.s larvae require approximately 10 days in sunlit habitats. However, it was revealed that tree canopy coverage exhibited a significant effect on the mosquito abundance in houses because it reduces the water temperature of larval habitats surrounding the houses because canopy cover reduces the amount of solar radiation reaching the larval habitats. It was also observed that the air temperature inside a house is affected by tree canopy.

Apart from the importance of congenial environmental and ecological factors such as breeding sites, humidity, temperature and rainfall, human activities such as agricultural practices, lumbering etc. also contribute to the distribution and abundance of these mosquito vectors especially the availability of host for blood meal. In conclusion, there was a high correlation of temperature and rainfall on the distribution and abundance of malaria and other related diseases associated with mosquito vectors. A small temperature rise either through seasonal variability, local microclimatic changes due to modification in vegetation cover or to global warming can increase disease transmission. The meteorological parameters are good prediction of malaria and associated disease risk. 


\section{Acknowledgement}

Authors are grateful to Bangladesh Meteorological Department, Abhawa Bhaban, for providing information on the study areas. Appreciation is expressed to National Science and Technology (NST) for providing fund. First author (MGS) is also grateful to Dean, Post Graduate Studies, Sher-e-Bangla Agricultural University, Dhaka for logistic support. Heartiest thanks to Mr. Kalam, Mr. Alamgir and Mr. Salam in the Department of Entomology, SAU and others who were of great help for carrying out the study.

\section{References}

Ahmed, S.M., R. Haque, U. Haque and A. Hossain. 2009. Knowledge on the transmission, prevention and treatment of malaria among two endemic populations of Bangladesh and their health-seeking behaviour. Malar J. 8: 173.

Briet, J., P. Vounatsou, D. Gunawardena, N. Galappaththy, and P. Amerasinghi. 2008. Temporal Correlation between Malaria and Rainfall in Sri Lanka. Malar J. 7: 77.

Craig, M., Snow and R. Le Sueur. 1999. A Climate based distribution model of malaria transmission in Sub Sahara Africa. Parasitol Today 15:105-111.

Giles, M. 1968. The Anopheline of Africa, South of the Saharan. No. 54 Johannesburg: The South African Institute for Medical Research 10-15.

Githeko, A. and W. Ndgwa. 2001. Predicting malaria epidemic in Kenya Highlands using climate data: A tool for decision maker. Glob Change Hum Health 2: 54-63.

Gubler, D., P. Reiter, K. Ebi. W. Yap, R. Nasci. and J. Patz. 2001. Climate variability and change in the United States: Potential impacts on vector - and rodent-borne diseases. Environ. Health Perspect 109: 22333.

Haque, U., R. Magalhaes, J. Reid, H. L. Clements, S. M. Ahmed and A. Islam. 2010. Spatial prediction of malaria prevalence in an endemic area of Bangladesh. Malar J. 9: 120.

Haque U, M. Hashizume, G. Glass, M. Ashraf, A. Dewan and H. Overjaard. 2007. The role of climate variability in the spread of malaria in Bangladesh highlands. PLoS One. 5(12): c14341.

Hashizume, M., T. Terao and N. Minakawa. 2009. The Indian Ocean Dipole and malaria risk in the highlands of Western Kenya. Proc. Natl. Acad. Sci. USA 106(6): 1857-1862.

Koekemoer, L., L. Kamau, R. Hunt and M. Coetzee. 2002. A Cocktail polymerase chain reaction (PCR) assay to identify members of the Anopheles funestus (Diptera: Culicidae) group. Am. J. Med. Hyg. 66: 804-811.

Lindsay, S.W. and M.H. Birley. 1996. Climate change and malaria transmission. Ann. Trop. Med. Parasitol. 90: 573-588.

Murty, U.S, S.R. Mutheneni and N. Arunachalam. 2010. The effects of climatic factors on the distribution and abundance of Japanese encephalitis vectors in Kurnool district of Andhra Pradesh, India. J. Vector Borne Dis. 47: 26-32.

Rogers, D.J. and S.E. Randolph. 2006. Climate change and vector-borne diseases. Adv. Parasitol. 62: $345-381$.

Strickland, C. and K. Choudhury. 1927. An illustration to the identification of the Anopheline larvae. Entomol. School Tropica Med. 2: 1-65.

Thomson, M., S. Mason, T. Phindela and S. Connor. 2005. Use of rainfall and sea surface temperature monitoring, for malaria early warning in Botswana. Am. J. Trop. Hyg. 73(1): 214-221. 
Thu N.C., T.R. Burkot. and B.H. Kay. 1998. The effects of temperature upon Aedes aegypti. Part I. The survival of Aedes aegypty eggs under abnormal temperature conditions. Am. J Hyg. 1932(3) 177-191.

WHO. 2009. World Health Malaria Report, Geneva, Switzerland.

WHO. 2010. Roll Back Malaria. Key Malaria Facts. Geneva, Switzerland.

WHO. 2012. The global malaria situation: current tools for prevention and control. Global fund to fight AIDS, tuberculosis and malaria. 55 world assembly, WHO, document no. A551. INF. Doc. 16.

Wu P.H. Guo, C. Lung. and H. Su. 2007. Weather as an effective predictor for occurrence of dengue fever in Taiwan. Acta. Tropica. 103: 50-57.

Zhou, G., N. Minakawa, K. Andrew, and Y. Guiyan. 2004. Association between climate variability and malaria epidemics in the East African Highlands. Proc. Nati Acad. Sci. 101: 2375-2380.

Zhou, G, Minakawa, K. Andrew and Y. Guiyan. 2007. Association between climate variability and dengue epidemics in the East African Highlands. Proc. Nati Acad. Sci. 110: 3475-3481.

(Revised copy received on 12.02.2020) 